\title{
Possibility of soft-matter effects in solids
}

\author{
H.V. Gomonay ${ }^{72}$, I.G. Kornienko², V.M. Loktev 1 \\ 1 Bogolyubov Institute for Theoretical Physics NAS of Ukraine, Metrologichna Str. 14-b, 03680, Kyiv, Ukraine \\ 2 National Technical University of Ukraine "KPI", Peremogy Avenue, 37, 03056, Kyiv, Ukraine
}

Received February 22, 2010

\begin{abstract}
Shape variation under the action of small external fields is a peculiar feature of soft matter. In the present paper we demonstrate a possibility of the analogous shape variation in the solids that combine the properties of antiferro- and ferromagnetic materials and show strong magnetoelastic coupling. The antiferromagnetic subsystem provides a macroscopic deformation of a sample in the external magnetic field while the ferromagnetic component ensures high susceptibility of the domain structure.
\end{abstract}

PACS: $75.60 . \mathrm{Ch}, 46.25 . \mathrm{Hf}, 75.50 . \mathrm{Ee}$

Key words: domain walls and domain structure, shape effects, thermoelasticity and electromagnetic elasticity, antiferromagnets

\section{Introduction}

It is of common knowledge that the solids, in contrast to soft matter materials, have a fixed, invariable shape. Variation of physical properties (including deformation) induced by external fields is described locally, in the thermodynamic limit, which excludes the sample boundaries from consideration. Soft matter (such as liquids, gels, etc.), as usual, has got fixed local properties (invariable in the presence of external fields) but can easily change the shape under the effect of thermal stresses or thermal fluctuations. However, the divide between the soft and "hard" matter is rather conditional because some solids can also change their shape without variation of the sample volume and local characteristics (like magnetization, polarization, etc). Good example of such shape-flexible systems is given by the nano-sized particles of antiferromagnetic (AFM) or martensitic materials.

The materials of these types show noticeable spontaneous deformations coupled with the primary order parameter (related to spin or charge distribution). Moreover, equilibrium states are usually realized in a set of different but equivalent configurations (domains) with the different strain tensors. The shape of the sample then depends on the domain structure (DS) and in many cases can be easily changed by small external fields.

While the physical mechanism of the DS formation is related with the sample boundary, reconstruction and restructurization of the domains under external fields depend upon the properties of the domain walls. If the potential barrier for the domain wall formation is high, the behaviour of the sample is solid-like, the switching between different macroscopic states is sharp, and field dependence of macroscopic properties shows a hysteresis. In an opposite case of low potential barrier, reconstruction of the DS takes place through nucleation and growth of new domains and shows the features of liquid-like behaviour: nonhysteretic transitions between different macroscopic states, shape deformation, etc. The most interesting case on which we concentrate our attention in the present paper, lies in-between: i.e., in multiferroics with the domains of different nature some types of domains can easily nucleate and show soft-like behaviour while the others could have high nucleation barrier and reveal themselves as solids. In particular, we consider a multiferroic (like $\mathrm{Sr}_{2} \mathrm{Cu}_{3} \mathrm{O}_{4} \mathrm{Cl}_{2}$ or $\mathrm{Ba}_{2} \mathrm{Cu}_{3} \mathrm{O}_{4} \mathrm{Cl}_{2}$ ) that simultaneously shows ferromagnetic (FM) and AFM ordering on different systems of copper ions [1].

\footnotetext{
*E-mail: malyshen@ukrpack.net
} 
The DS in such a material is characterized by two independent (FM and AFM) order parameters. Though macroscopic state of both FM and AFM could be controlled by the same, magnetic, field, the responses of the FM and AFM domain structures are different, as illustrated in figure 1 . The domain structure of FMs reconfigures in the magnetic field which is parallel to magnetiza-
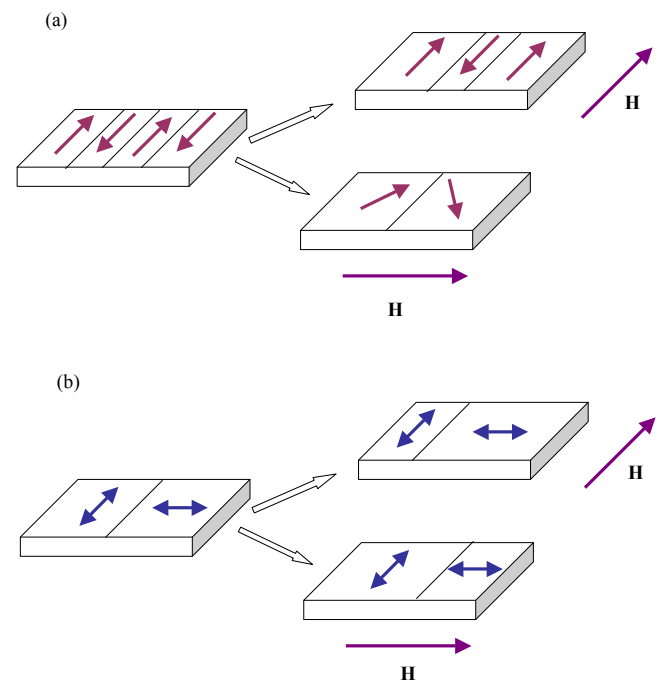

Figure 1. Behaviour of the FM (a) and AFM (b) DS in the external magnetic field $\mathbf{H}$. In the absence of field both types of domains (shown by arrows) are equally represented. (a) FM domains have opposite direction of magnetization vector. The magnetic field which is parallel to an easy axis (upper panel) removes degeneracy of the domains. As a result, the fraction of favorable domain increases. If $\mathbf{H}$ is perpendicular to easy axis, both types of domains are equivalent, the domain fraction does not change, the magnetic field induces tilt of magnetizations (lower panel). (b) AFM domains have different (perpendicular) orientation of AFM vectors. Degeneracy of domains is removed for any of two mutually perpendicular orientations of magnetic field.

tion and does not change if the magnetic field $\mathbf{H}$ is perpendicular to magnetization. In any case, switching between different domain states or smooth variation of the DS has an effect on macroscopic magnetization of the sample that grows linearly with the field value. The shape of the sample remains invariant. In contrast, the DS in antiferromagnets reconfigures for both (mutually perpendicular) orientations of the magnetic field and gives rise to variation of macroscopic deformation (and, hence, the shape) of the sample proportional to $H^{2}$. So, a material that at the same time bears the features of FM and AFM can show some new type of behaviour governed by competition between the domains of different nature in the external magnetic field.

In the present paper we analyze the physical mechanisms of soft-like behaviour in a multiferroic that combines shape softness of AFMs and magnetic softness of FMs.

We dedicate this paper to the 50-th anniversary of a well-known Ukrainian physicist Prof. I.M. Mryglod who greatly contributed into the field of soft-matter and, in particular, into the theory of magnetic liquids and whose results have deepened our understanding of corresponding systems.

\section{Shape softness induced by magnetoelastic interactions}

In FM and in ferroelectrics, the shape of the sample governs an equilibrium DS but is almost unchanged under the field effect because $i$ ) the domains with opposite magnetization/polarization have the same strain tensor; $i i)$ magnetoelastic coupling in these materials is usually much weaker compared to AFMs and martensites. On the contrary, in ferroelastic materials (such as martensites and AFMs with the pronounced magnetoelastic coupling) the shape of the sample is, in a certain 
sense, a free thermodynamic variable that can be adjusted by application of external fields. In this Section we present a general approach to a consideration of shape-related effects in AFMs.

Let us consider a tetragonal easy plane AFM in which AFM ordering is accompanied by the appearance of magnetoelastic strains. Equilibrium orientation of AFM vector $\mathbf{L}$ and deformations described by the strain tensor $\hat{u}$ could be found by minimization of the free energy $\Phi$ of the sample. In the finite-size sample the potential $\Phi$ includes the bulk- $(b)$ and the surface- $(S)$ dependent terms and, in the simplest case, (in neglection of a spin-dependent surface tension) it could be written as follows:

$$
\Phi=\int_{V}\left[f^{(\mathrm{mag})}(\mathbf{L})-\hat{u}: \hat{\lambda}: \mathbf{L} \otimes \mathbf{L}+\frac{1}{2} \hat{u}: \hat{c}: \hat{u}\right] \mathrm{d} V+\frac{1}{2} \oint_{S}\left\{K^{(S)}\left[\left(\mathbf{L} \cdot \tau_{1}\right)^{2}+\left(\mathbf{L} \cdot \tau_{2}\right)^{2}\right]\right\} \mathrm{d} S
$$

Here $f^{(\mathrm{mag})}(\mathbf{L})$ is the density of magnetic anisotropy energy within the bulk of the sample, the 4-th order tensors $\hat{\lambda}$ and $\hat{c}$ are magnetoelastic and elastic constants, respectively, $V$ is the sample volume. The last term in equation (11) is the surface energy that originates from the difference in the atomic environment in the bulk and at the surface. Vectors $\tau_{1,2}$ are space-dependent unit vectors tangential to the sample surface $S$.

Spatial distribution of $\mathbf{L}(\mathbf{r})$ and a displacement vector $\mathbf{u}(\mathbf{r})$ are then calculated from the set of differential equations

$$
\frac{\delta f^{(\mathrm{mag})}}{\delta \mathbf{L}}=\mathbf{L} \cdot \hat{\lambda}: \nabla \otimes \mathbf{u}, \quad \nabla \cdot \hat{c}: \nabla \otimes \mathbf{u}=\nabla \cdot \hat{\lambda}: \mathbf{L} \otimes \mathbf{L}
$$

with the (nontrivial) boundary conditions

$$
K^{(S)}\left[\tau_{1}\left(\mathbf{L}^{(\mathrm{S})} \cdot \tau_{1}\right)+\tau_{2}\left(\mathbf{L}^{(\mathrm{S})} \cdot \tau_{2}\right)\right]=0, \quad \mathbf{n} \cdot \hat{\hat{c}}:\left.\nabla \otimes \mathbf{u}\right|_{S}=\mathbf{n} \cdot \hat{\hat{\lambda}}: \mathbf{L}^{(\mathrm{S})} \otimes \mathbf{L}^{(\mathrm{S})}
$$

where $\mathbf{n}$ is a surface $S$ normal at a given point $\mathbf{r}_{S}$.

Analysis of equations (2) and (3) shows that for an arbitrary orientation of sample faces (i.e., vectors $\tau_{1,2}$ ) equilibrium orientations of $\mathrm{AFM}$ vector within the bulk $\left(\mathbf{L}^{(\mathrm{b})}\right)$ and at the surface $\left(\mathbf{L}^{(\mathrm{S})}\right)$ are different, $\mathbf{L}^{(\mathrm{b})} \neq \mathbf{L}^{(\mathrm{S})}$. This means that an $\mathrm{AFM}$ vector $\mathbf{L}$ and, as a result, a strain tensor $\hat{u}$ are space dependent functions. Due to the long-range character of the elastic forces this gives rise to the formation of equilibrium DS consistent with the shape of the sample.

To elucidate this point let us suppose that space dependence of $\mathbf{L}(\mathbf{r})$ is known. In this case the second of equations (2) for the displacement vector $\mathbf{u}$ together with the boundary conditions (3) is treated as a Neuman problem. Once Green function $\hat{G}\left(\mathbf{r}, \mathbf{r}^{\prime}\right)$ of an operator $\nabla \cdot \hat{c} \cdot \nabla$ is known, an equilibrium tensor of magnetoelastic strains can be presented as a symmetrized (sym) combination of the distortion, namely,

$$
\hat{u}(\mathbf{r})=\hat{\lambda}:\left(\mathbf{L}^{(\mathrm{b})} \otimes \mathbf{L}^{(\mathrm{b})}\right)+\operatorname{sym} \oint_{S}\left[\mathbf{L}^{(\mathrm{S})} \otimes \mathbf{L}^{(\mathrm{S})}-\mathbf{L}^{(\mathrm{b})} \otimes \mathbf{L}^{(\mathrm{b})}\right]: \hat{\lambda} \cdot \nabla \cdot \hat{G}\left(\mathbf{r}, \mathbf{r}^{\prime}\right) \mathrm{d} S^{\prime} .
$$

The first term in (4) is a standard magnetostriction that usually arises in the thermodynamic limit (when the surface effects are ignored). It can have different values if $\mathbf{L}^{(\mathrm{b})}$ is degenerated (magnetic anisotropy allows the states with different, noncollinear orientations of AFM vector). The second term, which is generally nonzero, depends upon the shape of the sample. It represents an additional strain induced by the surface "magnetoelastic charges". If the surface curvature is not too large (characteristic scale of $\tau_{1,2}$ and $\mathbf{L}^{(\mathrm{S})}$ variation is much larger than the magnetic inhomogeneuity length), then, equilibrium magnetic structure of the sample consists of domains that in average compensate the field of magnetoelastic "charges". Equilibrium shape of the sample can be characterized by an average deformation which in this particular case is equal to

$$
\langle\hat{u}\rangle=\hat{\lambda}:\left\langle\mathbf{L}^{(\mathrm{b})} \otimes \mathbf{L}^{(\mathrm{b})}\right\rangle \equiv \sum_{j} \hat{\lambda}: \mathbf{L}_{j}^{(\mathrm{b})} \otimes \mathbf{L}_{j}^{(\mathrm{b})} \xi_{j}
$$


where $\xi_{j}$ is the volume fraction of the domain of $j$ type.

Figure 2 illustrates some possible shape effects induced by the surface energy. In the magnetically homogeneous sample (figure 2(a)), the preferable orientation of AFM vector at the surface, $\mathbf{L}^{(\mathrm{S})}$, differs from $\mathbf{L}^{(\mathrm{b})}$ at the "top" and "bottom" faces and sets conditions for the formation of "magnetoelastic charges" at these faces (see the second term in (4)). Corresponding stress field can be relaxed in two ways: $i$ ) by homogeneous deformation (and corresponding shape variation) if the sample size is of the order of magnetic inhomogeneity, figure 2 (b); ii) by forming a fine DS that compensates the stress field in average, figure 2 (c). Restructurization of the DS in the external magnetic field is related with the shape variation, as shown in figure 2 (d).

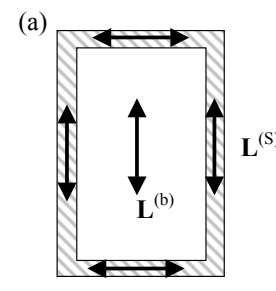

(c)

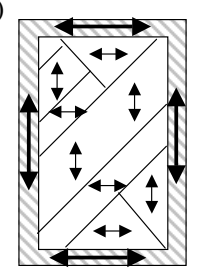

(b)

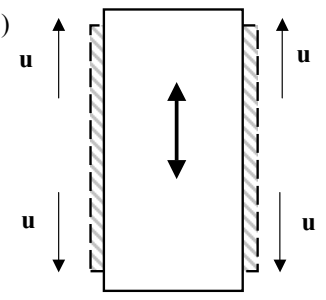

(d)

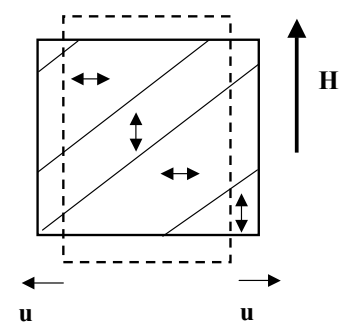

Figure 2. Destressing effects in an AFM of rectangular shape. (a) Single domain sample. Equilibrium orientation of the bulk, $\mathbf{L}^{(\mathrm{b})}$, and the surface, $\mathbf{L}^{(\mathrm{S})}$, AFM vectors are different at some faces. Shaded area shows surface regions. (b) Misfit between $\mathbf{L}^{(\mathrm{b})}$ and $\mathbf{L}^{(\mathrm{S})}$ induces elongation of "favourable" (shown with arrows) and contraction of "unfavourable" faces. Orientation of AFM vector at the surface (not shown) is the same as in (a). (c) DS eliminates the misfit between $\mathbf{L}^{(\mathrm{b})}$ and $\mathbf{L}^{(\mathrm{S})}$ in average. The shape of the sample is not changed. (d) External magnetic field gives rise to restructurization of the DS and, as a consequence, to shape variation.

An effective formalism for the description of the DS in this case is based on consideration of the destressing energy [2]

$$
\Phi^{\text {dest }}=\frac{V}{2}\langle\mathbf{L} \otimes \mathbf{L}\rangle: \hat{\lambda}: \hat{\aleph}: \hat{\lambda}:\langle\mathbf{L} \otimes \mathbf{L}\rangle,
$$

where the brackets $\langle\cdots\rangle$ mean averaging over the sample volume, as in equation (5). The components of the destressing tensor $\hat{\aleph}$

$$
\hat{\aleph} \equiv \nabla \otimes \nabla \int_{V} \hat{G}\left(\mathbf{r}-\mathbf{r}^{\prime}\right) \mathrm{d} V^{\prime}
$$

depend upon the shape of the sample and anisotropy of the elastic forces.

In a particular case of tetragonal crystal cutted in a form of an ellipsoid (semiaxes $a>b \gg c$ ) whose principal axes $X, Y$ are parallel to an AFM easy plane (see figure 4), the destressing energy (6) takes a form :

$\Phi^{\text {dest }}=\frac{V}{2}\left\{N_{2}^{\text {des }}\left\langle L_{X}^{2}-L_{Y}^{2}\right\rangle+N_{\text {is }}^{\text {des }}\left[\left\langle L_{Y}^{2}-L_{X}^{2}\right\rangle^{2}+4\left\langle L_{X} L_{Y}\right\rangle^{2}\right]-N_{4 a n}^{\text {des }}\left[\left\langle L_{X}^{2}-L_{Y}^{2}\right\rangle^{2}-4\left\langle L_{X} L_{Y}\right\rangle^{2}\right]\right\}$. 
An explicit form of the effective shape-induced anisotropy constants $N^{\text {des }}$ depends upon the elastic and magnetoelastic properties of the crystal which we assume to be isotropic (that means, in particular, the following relation between the elastic modula: $c_{11}-c_{12}=2 c_{44}$ ). Then [2],

$$
N_{\mathrm{is}}^{\mathrm{des}}=\frac{\lambda^{2}(3-4 \nu)}{8 c_{44}(1-\nu)}, \quad N_{2}^{\mathrm{des}}=\frac{c}{b} \cdot \frac{\left[\lambda^{2}(2-3 \nu)+\lambda_{v} \lambda\right] J_{2}(k)}{4 c_{44}(1-\nu)}, \quad N_{4 \mathrm{an}}^{\mathrm{des}}=\frac{c}{b} \cdot \frac{2 \lambda^{2} J_{4}(k)}{3 c_{44}(1-\nu)},
$$

where $\lambda, \lambda_{v}$ are magnetoelastic constants, $c_{44}$ is the shear modulus, $\nu=c_{12} /\left(c_{11}+c_{12}\right)$ is the Poisson ratio and we have introduced the dimensionless shape-factors $J_{2,4}(k)$ as follows

$$
J_{2}(k)=\int_{0}^{\pi / 2} \frac{\left(\sin ^{2} \phi+\cos 2 \phi / k^{2}\right) \mathrm{d} \phi}{\sqrt{1-k^{2} \sin ^{2} \phi}}, \quad J_{4}(k)=\int_{0}^{\pi / 2} \frac{\left(1-8 \cos 2 \phi-k^{2} \sin ^{2} \phi+8 \cos 2 \phi / k^{2}\right) \mathrm{d} \phi}{\sqrt{1-k^{2} \sin ^{2} \phi}} .
$$

Here the parameter $k^{2}=1-b^{2} / a^{2}$ depends upon the aspect ratio $b / a$ of the sample.

In the next section we will show how to change an average deformation (5) and, correspondingly, the shape of multiferroic by application of small external field.

\section{Field-induced variation of the sample shape}

We consider the model FM+AFM multiferroic [3 [5] whose magnetic structure consists of two weakly coupled subsystems, AFM, and FM, localized on different types of magnetic ions CI and CII, as shown in figure 3. A FM subsystem is unambiguously described by a magnetization vector $\mathbf{M}_{\mathrm{F}}$ and an AFM subsystem is described by two vectors: an $\mathrm{AFM}$ vector $\mathbf{L}=\left(\mathbf{S}_{1}-\mathbf{S}_{2}+\mathbf{S}_{3}-\mathbf{S}_{4}\right) / 4$ and a $\mathrm{FM}$ vector $\mathbf{M}=\sum_{j} \mathbf{S}_{j} / 4$.

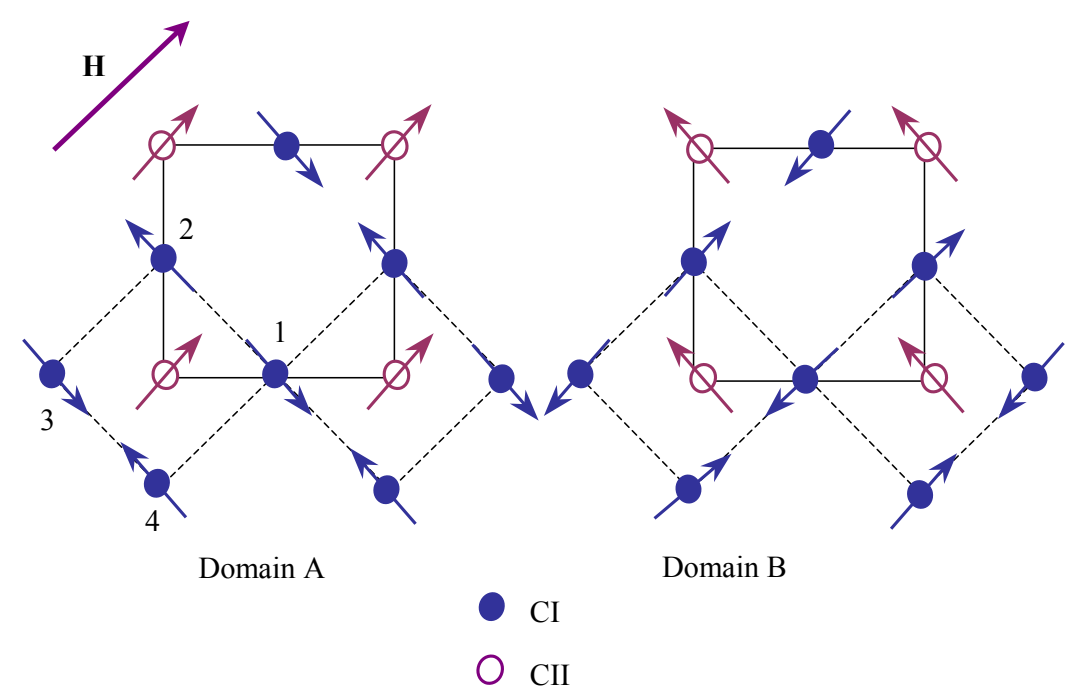

Figure 3. Magnetic structure of a square lattice in two domain configurations. Magnetic field is parallel to $\langle 110\rangle$. Two types of magnetic ions are represented with the filled and hollow circles. The FM ordered moments of CII could be (a) parallel (domain A) or (b) perpendicular (domain B) to the applied magnetic field. Small canting of the CI spins induced by the external magnetic field is not shown.

In the absence of the external field, the FM moments at CII sites are oriented along $\langle 110\rangle$ crystal directions perpendicular to the staggered magnetizations of AFM subsystem, as shown in figure 3. Due to tetragonal symmetry of the crystal, an equilibrium magnetic structure can be realized in four types of equivalent domains (figures 3 and 4). Types A and B could be thought of as AFM domains because they correspond to different orientations of $\mathbf{L}$ vector and thus are sensitive to the orientation of magnetic field $\mathbf{H}$ with respect to crystal axes (see figure 1). Types 
A1 and A2 (and, correspondingly, B1 and B2) are FM domains. They have opposite direction of $\mathbf{M}_{\mathrm{F}}$ vector and could be removed from the sample by $\mathbf{H} \| \mathbf{M}_{\mathrm{F}}$.
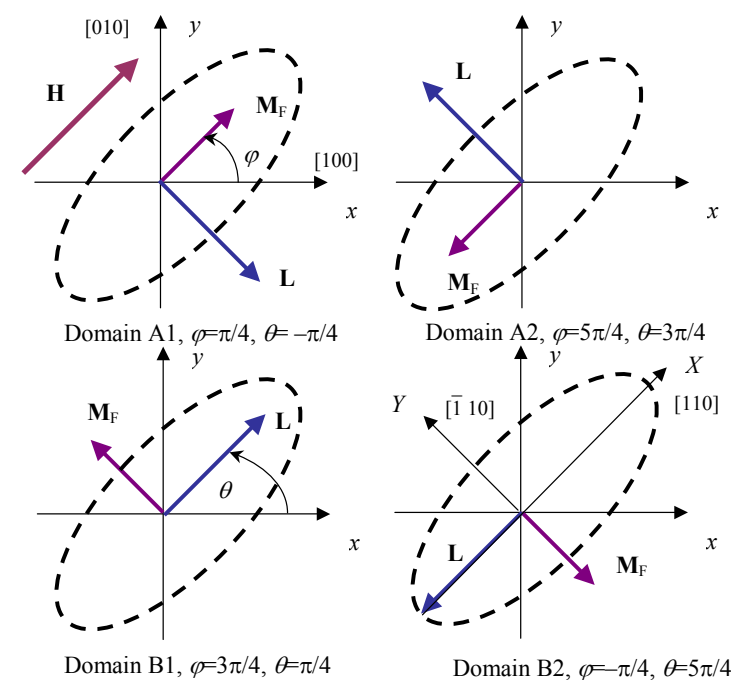

Figure 4. Four types of magnetic domains. Axes $x$ and $y$ are parallel to $\langle 100\rangle$ crystal directions. An external magnetic field $\mathbf{H} \|[110]$ (if any). Types A and B have different orientations of AFM vector, types 1 and 2 correspond to opposite directions of $F M$ vector $\mathbf{M}_{\mathrm{F}}$. Ellipse (dash line) images the shape of the sample and its orientation with respect to crystal axes.

Phenomenological description of the DS is based on the analysis of free energy potential $\Phi$ that consists of three terms: the bulk magnetic energy $\Phi_{\text {mag }}$, the destressing energy $\Phi_{\text {dest }}$ (see equation (88) that implicitly includes the surface contribution, and stray (demagnetizing) energy $\Phi_{\text {stray }}$,

$$
\Phi=\Phi_{\text {mag }}+\Phi_{\text {dest }}+\Phi_{\text {stray }} .
$$

Following the papers $[1,4,[4]$ we take the magnetic energy in a form:

$$
\Phi_{\mathrm{mag}}=\int_{V} \mathrm{~d} V\left\{\frac{4}{M_{0}^{2}}\left[2 J_{0} \mathbf{M}^{2}+J_{\mathrm{av}} \mathbf{M} \cdot \mathbf{M}_{\mathrm{F}}+J_{\mathrm{pd}} \mathbf{M}_{\mathrm{F}} \hat{\sigma}_{z} \mathbf{L}\right]-\frac{8}{M_{0}^{4}} K_{\|} L_{x}^{2} L_{y}^{2}-\mathbf{H} \cdot \mathbf{M}_{\mathrm{F}}-2 \mathbf{H} \cdot \mathbf{M}\right\} .
$$

Here $M_{0}$ is CI sublattice magnetization, orthogonal axes $x$ and $y$ are parallel to the crystal directions [100] and [010], respectively. $J_{0}$ is an exchange constant responsible for the AFM ordering, $J_{\text {av }}$ and $J_{\mathrm{pd}}$ describe, respectively, isotropic and anisotropic pseudodipolar interactions between the FM and AFM subsystems, $K_{\|}$is in-plane anisotropy, $\hat{\sigma}_{z}$ is a Pauli matrix.

For an elliptic-shaped sample, the stray energy can be written as follows:

$$
\Phi_{\text {stray }}=\frac{V}{2}\left[N_{a}^{\mathrm{dm}}\left\langle M_{\mathrm{FX}}+2 M_{X}\right\rangle^{2}+N_{b}^{\mathrm{dm}}\left\langle M_{\mathrm{FY}}+2 M_{Y}\right\rangle^{2}\right] .
$$

The components of demagnetization tensor $N_{a, b}^{\mathrm{dm}}$ are calculated in a standard way [7]

$$
N_{a}^{\mathrm{dm}}=\frac{4 \pi c}{a \sqrt{1-k^{2}}} \int_{0}^{\pi / 2} \frac{\sin ^{2} \phi \mathrm{d} \phi}{\sqrt{1-k^{2} \sin ^{2} \phi}}, \quad N_{b}^{\mathrm{dm}}=\frac{4 \pi c \sqrt{1-k^{2}}}{a} \int_{0}^{\pi / 2} \frac{\cos ^{2} \phi \mathrm{d} \phi}{\sqrt{1-k^{2} \sin ^{2} \phi}} .
$$

Equations (12), (13), and (8) could be substantially simplified if we take into account that: i) far below the Néel temperature the sublattice magnetizations $M_{0}$ and $M_{\mathrm{F}}$ are constant; as a result, ii) $\mathbf{L} \perp \mathbf{M}$ and $\mathbf{L}^{2}+\mathbf{M}^{2}=M_{0}^{2}$ (normalization conditions); iii) if the magnetic field is much 
smaller than spin-flip field, $H \ll J_{0} / M_{0}$, the magnetization induced in AFM subsystem is small], $M \ll M_{0}$, and vector $\mathbf{M}$ can be excluded from (12) as follows [8]:

$$
\mathbf{M}=\frac{1}{8 J_{0}}\left[\mathbf{L} \times\left[\left(\mathbf{H}-2 \frac{J_{\mathrm{av}}}{M_{0}^{2}} \mathbf{M}_{\mathrm{F}}\right)\right]\right]
$$

$i v$ ) if an out-of-plane anisotropy is strong enough, all the magnetic vectors lie within $x y$ (and, equivalently, $X Y$ ) plane and could be described with the only angle variable, as shown in figure 4:

$$
L_{x}=M_{0} \cos \theta, \quad L_{y}=M_{0} \sin \theta ; \quad M_{\mathrm{F} x}=M_{\mathrm{F}} \cos \varphi, \quad M_{\mathrm{F} y}=M_{\mathrm{F}} \sin \varphi .
$$

With account of the relations (15) and (16), the free energy (11) takes the following form

$$
\begin{aligned}
\Phi= & V\left\{\frac{4 M_{\mathrm{F}}}{M_{0}} J_{\mathrm{pd}}\langle\cos (\theta+\varphi)\rangle+K_{\|}\langle\cos 4 \theta\rangle-\frac{J_{\mathrm{av}}^{2} M_{\mathrm{F}}^{2}}{8 J_{0} M_{0}^{2}}\langle\cos 2(\theta-\varphi)\rangle-\frac{J_{\mathrm{av}}}{8 J_{0}} H M_{\mathrm{F}}\langle\cos (2 \theta-\psi-\varphi)\rangle\right. \\
& -H M_{\mathrm{F}}\left(1-\frac{J_{\mathrm{av}}}{8 J_{0}}\right)\langle\cos (\varphi-\psi)\rangle+\frac{H^{2} M_{0}^{2}}{32 J_{0}}\langle\cos 2(\theta-\psi)\rangle-\frac{1}{2} N_{2}^{\mathrm{des}}\langle\cos 2(\theta-\psi)\rangle \\
& +\frac{1}{2} M_{\mathrm{F}}^{2}\left[N_{a}^{\mathrm{dm}}\langle\cos (\varphi-\psi)\rangle^{2}+N_{b}^{\mathrm{dm}}\langle\sin (\varphi-\psi)\rangle^{2}\right] \\
& \left.+\frac{1}{2}\left(N_{\mathrm{is}}^{\mathrm{des}}+N_{4 \mathrm{an}}^{\mathrm{des}}\right)\langle\cos 2(\theta-\psi)\rangle^{2}+2\left(N_{\mathrm{is}}^{\mathrm{des}}-N_{4 \mathrm{an}}^{\mathrm{des}}\right)\langle\sin 2(\theta-\psi)\rangle^{2}\right\} .
\end{aligned}
$$

where $\psi$ is an angle between magnetic field and $x$-axis, and we assume that the field is parallel to one of the principal axes of the sample.

For the rest of the paper we assume that the magnetic field is parallel to one of the easy axes, $\mathbf{H} \|[110]$, so, $\psi=\pi / 4$. In an infinite sample (all $N^{\mathrm{dm}}, N^{\mathrm{des}}=0$ ) minimization of $\Phi$ with respect to magnetic variables $\theta$ and $\varphi$ gives rise to the four solutions labeled as domains A1,2 and B1,2 (see figure (4). It should be stressed that in contrast to pure AFMs, the configurations with $\left(\mathbf{M}_{\mathrm{F}}, \mathbf{L}\right)$ and $\left(\mathbf{M}_{\mathrm{F}},-\mathbf{L}\right)$ are inequivalent, due to anisotropic pseudodipolar interactions (described by the constant $J_{\mathrm{pd}}$ ). Figure [5illustrates the field-induced variation of equilibrium magnetic configurations (represented by $X$-projections of $\mathbf{M}_{\mathrm{F}}$ and $\mathbf{L}$ vectors) obtained from the numerical minimization of expression (17) with the data taken from [1, 4, 6].

One can distinguish three field intervals. At small fields, $|H| \leqslant H_{\mathrm{s}-\mathrm{f} 1} \propto J_{\mathrm{pd}}$, the DS of the sample may include all four domain types. However, the energies $E_{j}(j=A 1,2, B 1,2)$ of the states are different: $E_{\mathrm{A} 1}<E_{\mathrm{B}}<E_{\mathrm{A} 2}$ (domains $\mathrm{B} 1$ and $\mathrm{B} 2$ are equivalent if $\mathbf{H} \|[110]$ ). In the interval $H_{\mathrm{s}-\mathrm{f} 1}<|H|<H_{\mathrm{s}-\mathrm{f} 2} \propto \sqrt{J_{0} K_{\|}}$the DS may consist of only FM domains, A1 and A2. At last, at $|H| \geqslant H_{\mathrm{s}-\mathrm{f} 2}$ the sample is a single domain (A1).

It can be seen from figure 5 (a) that the formation of AFM, B-type domain is energetically preferable than the formation of FM domain A2. So, for certain conditions, the DS structure of a sample may consist of the domains of only two types, A1 and B. This means that the field induces the variation of average deformation

$$
\left\langle u_{X X}-u_{Y Y}\right\rangle \propto\langle\cos 2(\theta-\psi)\rangle=\frac{|H| M_{0}}{m_{\mathrm{F}}\left(N_{\mathrm{is}}^{\text {des }}+N_{\text {4an }}^{\text {des }}\right)},
$$

and, as a result, variation of the shape. It is interesting that in contrast to pure AFMs, $\langle\cos 2(\theta-$ $\psi)\rangle \propto|H|$ (not to $H^{2}$ ). This nontrivial behaviour is due to the presence of FM subsystem. Really, relative fraction of $\mathrm{A} 1$ and $\mathrm{B}$ domains is proportional to the energy difference $E_{\mathrm{B}}-E_{\mathrm{A} 1}$. The main contribution into this difference arises from interaction of FM subsystem with the external magnetic field (Zeeman term $\mathbf{H M}_{\mathrm{F}}$ in equation (12)). Magnetic susceptibility of shape/deformation $(=\mathrm{d}\langle u\rangle / \mathrm{d} H)$ is inversely proportional to the destressing coefficient and can be much higher than

\footnotetext{
${ }^{1}$ In FM+AFM multiferroic we have an additional (compared to pure AFM) limitation, $J_{\mathrm{av}} M_{\mathrm{F}} \ll J_{0} M_{0}$, which means that the coupling between the FM and AFM subsystems should be weaker than the exchange interaction between AFM coupled sites.
} 

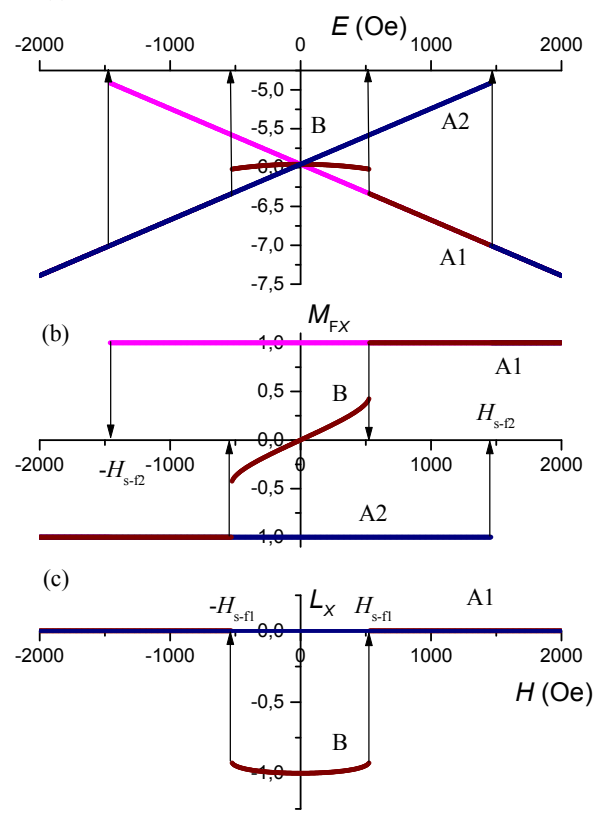

Figure 5. Stability ranges of homogeneous configuration shown in figure 4 in the external magnetic field $\mathbf{H} \|[110]$. (a) Energy of equilibrium homogeneous state vs $H$. (b), (c) Normalized projections of FM and AFM moments on the field direction. Field induces rather noticeable rotation of $\mathbf{M}_{\mathrm{F}}$ vector toward field direction (b) and slight tilt of $\mathbf{L}$ vector (c). Loss of stability takes place at critical values $H=H_{\mathrm{s}-\mathrm{f} 1,2}$, as shown with arrows.

in a pure AFM (where all the effects are enhanced due to the exchange coupling and $N^{\text {des }}$ should be substituted for $\sqrt{N^{\text {des } J_{0}}}$ ).

Thus, in the FM+AFM multiferroic, the shape variation can be induced by small (compared to pure AFMs) external magnetic field.

\section{Conclusions}

We have analyzed some possible shape effects in magnetoelastic materials and found a certain duality that makes these solids similar to soft matter. In particular, not only the shape of the sample affects its macroscopic parameters, such as magnetization, polarization, etc., but also the field-induced variation of macroscopic parameters can define the sample shape. Change of the shape may proceed by reconstruction of the DS. Susceptibility of the shape to the external fields can be increased by the use of multiferroics instead of pure ferroelastic materials. In particular, field dependence of the average deformation switches from quadratic (in pure AFMs) to linear (in FM+AFM multiferroics).

An analogous treatment can be applied to multiferroics that combine electric polarization with AFM ordering. Soft-like behaviour of these materials (if any) may open new ways to control the shape of the sample with the electric field.

\section{Acknowledgements}

This work was partially supported by the Special Programme of Fundamental Research of the Department of Physics and Astronomy of National Academy of Science, Ukraine. 


\title{
References
}

1. Kastner M.A., Aharony A., Birgeneau R.J., Chou F.C., Entin-Wohlman O., Greven M., Harris A.B., Kim Y.J., Lee Y.S., Parks M.E., Zhu Q., Phys. Rev. B, 1999, 59, 14702-14711.

2. Gomonay H.V., Loktev V.M., Phys. Rev. B, 2007, 75, 174439.

3. Noro S., Kouchi T., Harada H., Yamadaya T., Tadokoro M., Suzuki H., Mater. Sci. Eng. B, 1994, 25, $167-170$.

4. Kim Y.J., Birgeneau R.J., Chou F.C., Greven M., Kastner M.A., Lee Y.S., Wells B.O., Aharony A., Entin-Wohlman O., Korenblit I.Y., Harris A.B., Erwin R.W., Shirane G., Phys. Rev. B, 2001, 64, 024435 .

5. Parks B., Kastner M.A., Kim Y.J., Harris A.B., Chou F.C., Entin-Wohlman O., Aharony A., Phys. Rev. B, 2001, 63, 134433.

6. Chou F.C., Aharony A., Birgeneau R.J., Entin-Wohlman O., Greven M., Harris A.B., Kastner M.A., Kim Y.J., Kleinberg D.S., Lee Y.S., Zhu Q., Phys. Rev. Lett., 1997, 78, 535-538.

7. Akhiezer A.I., Bar'yakhtar V.G., Peletminskii S.V., Spin Waves, vol. 1 of North-Holland Series in Low Temperature Physics. North-Holland, Interscience (Wiley) edn., Amsterdam, 1968.

8. Kosevich A.M., Ivanov B.A., Kovalev A.S., Nonlinear Magnetization Waves. Dynamical and Topological Solitons. Naukova dumka, Kiev, 1983.

\section{Про можливість ефектів м'якої матерії в твердих тілах}

\author{
О.В. Гомонай12, Є.Г. Корнієнко \\ 1 ІТФ НАН України ім. М.М. Боголюбова, вул. Метрологічна 14-б, 03680, Київ, Україна \\ 2 Національний технічний університет України “Київський політехнічний інститут”, \\ пр. Перемоги, 37, 03056, Київ, Україна
}

Характерною рисою м'якої матерії є їі властивість змінювати форму під впливом слабких зовнішніх полів. В роботі показана можливість аналогічної зміни форми в твердих тілах, які поєднують властивості антиферо- та феромагнітних матеріалів та мають сильний магнітопружний зв'язок. Макроскопічні деформації зразка в зовнішньому магнітному полі виникають за рахунок антиферомагнітної складової, а феромагнітна компонента обумовлює високу сприйнятливість доменної структури.

Ключові слова: доменні стінки та доменна структура, ефекти форми, термопружність та електромагнітна пружність, антиферомагнетики 
